\title{
THE STRIP METHOD OF NOISE-IMMUNE IMAGE TRANSFORMATION
}

\author{
L. A. Mironovskii ${ }^{1}$ and V. A. Slaev ${ }^{2}$
}

UDC 389.681.2

\begin{abstract}
Various methods are considered for image strip transformation, and the best transformation matrices are selected, with a definition of the critical multiplicity of the noise and studies on the root images for one-sided and two-sided strip transformation, and an illustration is given of the scope for this method in the strip transformation of a particular image.
\end{abstract}

Key words: image strip transformation, optimal transformation matrices, critical noise multiplicity, root images for one-sided and two-sided strip transformation.

Many tasks in information transformation and data analysis involve processing and transmitting images; examples are the scanning and analysis of the Earth's surface from satellites, x-radiography and its uses in medicine, and research on biological and chemical processes. The accuracy of the results is dependent on the image quality.

Here we examine the strip method for storage and noise-immune transmission of images, which had been proposed previously for processing one-dimensional signals [1,2]. Use is made of matrix transformations of the initial image before transmission, in which image fragments are mixed and superimposed. The transformed image is transmitted over a communication channel corrupted by pulse noise. It may result for example in complete loss of some image fragments. The signal at the receiving end is subjected to the inverse transformation, which yields image reproduction. If one provides a uniform distribution of the pulse noise over the entire image area (without change in energy), then there is an appreciable weakening of the noise amplitude and an acceptable quality will be attained in all parts of the recovered image.

The tasks of this article are as follows: to consider various forms of strip transformation, choose the best transformation matrices, determine the critical noise multiplicity, examine root strip transformation images, and illustrate the scope for the method on a particular example.

Two-Dimensional Strip Transformation. The first stage in the strip method for transforming one-dimensional signals $[1,2]$ consists in dividing up the initial signal into $n$ parts of identical length and generating from them an $n$-dimensional vector $X$. In the second stage, that vector is used in isometric transformation by multiplication by an orthogonal matrix $A$ of dimensions $n \times n: Y=A X$.

Similarly, the first stage in the strip transformation of a two-dimensional signal (image) consists in splitting up the initial image $P$ into $N$ rectangular fragments identical in size. We denote by $m$ and $n$ the numbers of horizontal and vertical strips into which the image is dissected; then $N=m n$.

Then a linear combination of the fragments is performed. Two approaches are possible: vector and matrix ones.

In the first (vector approach), the fragments are used in generating a $N$-dimensional block vector $X$, which as in the onedimensional case is subject to isometric transformation by multiplication by an orthogonal matrix $A$ of $\operatorname{dimensions} N \times N$ : $Y=A X$.

\footnotetext{
${ }^{1}$ St. Petersburg State University of Aviation Instrumentation; e-mail: mir@aanet.ru.

2 Mendeleev All-Russia Metrology Research Institute; e-mail: V.A.Slaev@vniim.ru.
}

Translated from Izmeritel'naya Tekhnika, No. 8, pp. 6-12, August, 2006. Original article submitted April 25, 2006. 
Then this vector after defragmentation is transmitted over the communication channel. We call this one-sided strip transformation as it is completely analogous to the one-dimensional case. The main shortcomings are the excessively high dimensions of matrix $A$ and the consequent related major computing exercise.

In the second (matrix method), the initial image is split up into fragments, which are considered as a block matrix $X$ of dimensions $m \times n$. Here there are three modes of isometric transformation to the mixed fragments:

a) multiplication by an orthogonal $m \times n$ matrix $B$ from the left: $Z_{1}=B X$ (left-sided matrix transformation);

b) multiplication by an orthogonal $n \times n$ matrix $A$ from the right: $Z_{2}=X A$ (right-sided matrix transformation); and

c) simultaneous multiplication by matrix $B$ from the left and by matrix $A$ from the right: $Z_{3}=B X A$ (two-sided matrix transformation).

All these cases are represented in Fig. 1, which displays the transformation chain for the initial image $P$, whose result is an image transmitted over a communication channel.

Particular interest attaches to the first and last forms of transformation, since they provide the fullest mixing of the image fragments, and each fragment of the transformed image contains information about all $m n$ fragments of the initial image $P$. In the other two cases $Z_{1}=B X$ and $Z_{2}=X A$, there is linear combination only of horizontal strips or only vertical ones in the initial image.

Then in what follows we will consider only two forms of transformation:

1) one-sided strip transformation

$$
Y=A X
$$

where $X$ is a block vector of dimensions $m n \times 1$, and $A$ is an orthogonal matrix of order $m n$;

2) two-sided strip transformation

$$
Z=B X A,
$$

where $X$ is a block matrix of dimensions $m \times n$, while $B$ and $A$ are orthogonal matrices of orders $m$ and $n$, respectively.

Correspondingly, the inverse transformations performed on restoring the image at the receiving end are described by

$$
X=A^{-1} Y
$$

for one-sided transformation and

$$
X=A^{-1} Z B^{-1}
$$

for two-sided transformation.

We now describe these cases in more detail.

Image transmission with one-sided strip transformation involves the assumption that the symbols $P$ and $P^{\prime}$ denote the initial and restored images, while $S$ and $S^{-1}$ are the forward and reverse strip operators, which perform the fragmentation and defragmentation.

To the vector $Y=A X$ in the communication channel one adds pulse interference $\Delta$, so the output is the image vector $Y^{\prime}=Y+\Delta$; at the receiving end, one performs the inverse one-sided strip transformation to get the vector $X^{\prime}$, which is described by

$$
X^{\prime}=A^{-1} Y^{\prime}=A^{-1}(Y+\Delta)=A^{-1} Y+A^{-1} \Delta=X+A^{-1} \Delta .
$$

This vector $X^{\prime}$ is the sum of the vectors $X$ and the noise vector $\Delta$, on which one performs the inverse transformation. In the last stage, the vector $X^{\prime}$ is transformed to a matrix of dimensions $m \times n$, which describes the reconstructed image $P$ with the addition of noise $\Delta^{\prime}=A^{-1} \Delta$.

The main shortcoming of one-sided strip transformation is the excessive size of the matrix $A$, which is $m n \times m n$ (the number of elements in this matrix is equal to the square of the number of fragments into which the image is divided). 


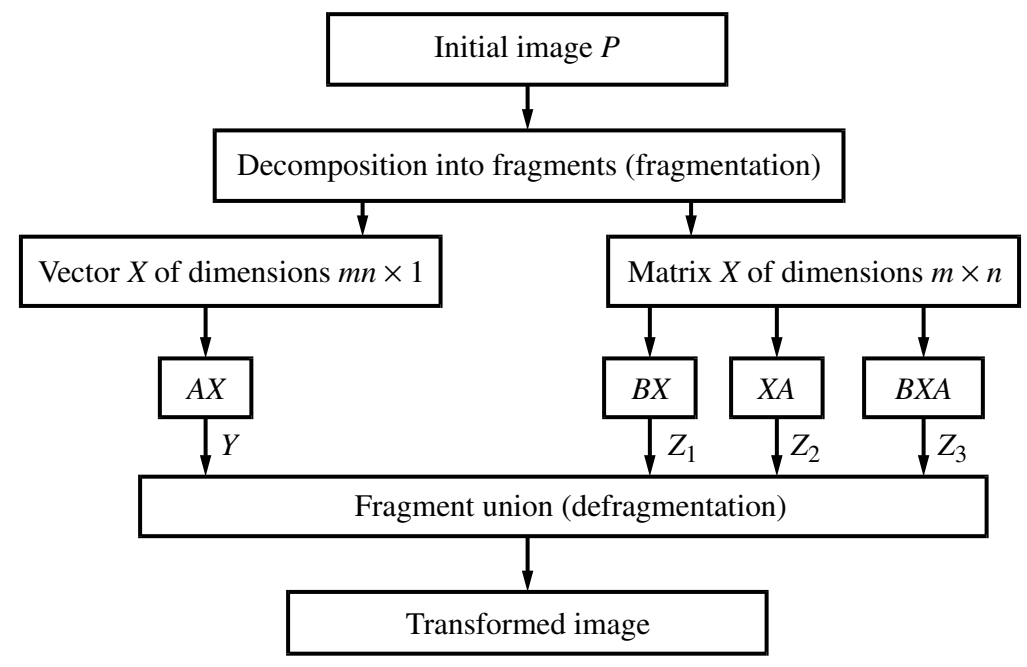

Fig. 1. Forms of two-dimensional strip transformation.

The matrices $B$ and $A$ used in two-sided strip transformation are of substantially smaller dimensions (with $m=n$, the total numbers of their elements are equal to twice the number of image fragments). This facilitates their generation and storage.

When an image is transmitted by two-sided strip transformation, the communication channel receives the image $Z=B X A$ derived by two-sided strip transformation of the initial image $P$. In the channel, pulse noise $\Delta$ is added (block matrix of dimensions $m \times n$ ), and so at the output of the channel we have the image $Z^{\prime}=Z+\Delta$. At the receiving end, $Z^{\prime}$ is subject to inverse two-sided transformation to obtain the matrix for the resultant image $P^{\prime}$. It is described by

$$
P^{\prime}=A^{-1} Z^{\prime} B^{-1}=A^{-1}(Z+\Delta) B^{-1}=A^{-1} Z B^{-1}+A^{-1} \Delta B^{-1}=P+A^{-1} \Delta B^{-1} .
$$

In accordance with (6), the recipient sees the initial image $P$ with the noise added to it in the channel, to which the inverse two-sided strip transformation has been applied.

It is convenient to take matrices $A$ and $B$ as equal because this simplifies the calculations and saves memory; then (2) becomes

$$
Z=A X A,
$$

where $A$ is an orthonormalized matrix.

Equation (6) also simplifies:

$$
P^{\prime}=P+A^{\mathrm{T}} \Delta A^{\mathrm{T}}
$$

To simplify the transformation further, it is convenient to use a symmetrical matrix $A$. Then the inverse transformation coincides with the forward one and there is no need to store separately or calculate the inverse matrix. The noise at the output will be expressed by $\Delta^{\prime}=A \Delta A$.

In (1)-(5) and other formulas in this section, one multiplies the ordinary numerical matrices $A$ and $B$ by the block matrices $X, Y$, and $Z$, whose elements are image fragments. The following rules are used:

1. Block addition (fragments). The individual blocks (fragments) of the image matrices are obtained by summing the corresponding block elements. This operation is analogous to adding together two matrices of identical size.

2. Multiplying a fragment by a number. The operation is performed by multiplying each pixel in a fragment by the number. This alters the brightness of the fragment as a whole. The operation is analogous to multiplying a matrix by a number. 
3. Multiplying a block matrix by a numerical matrix. That multiplication is performed in the same way as the ordinary multiplication of numerical matrices on the row by column rule on the basis of using the first two operations.

The above relates to black-and-white images. They are represented as matrices, in each case consisting of individual pixels (brightness values). Such a matrix is subject to fragmentation in strip transformation. The situation is somewhat more complicated for color images. One of the standard methods of representing the latter is a three-layer RGB matrix. Each pixel is encoded with the brightness values for red, green, and blue, and in that case the strip transformation is applied separately for each of the three layers.

Choice of Optimal Transformation Matrices. The fragmentation gives a block matrix containing $m \times n$ blocks, whose elements are rectangular and have dimensions $x \times y$. All fragments have the same size. When the number of pixels in a row or column for the initial image matrix does not divide without remainder by $m$ or $n$, it is necessary to add a pixel to the right or below in the image. They should not distort or excessively alter the contrast of the image.

The fragmentation considerably reduces the computation volume by comparison with elementwise processing; the larger the fragments, the less the size of matrix $A$. The size of an image fragment should be chosen on the basis of the expected scale of the pulse noise, i.e., the linear dimensions of the corrupted part of the image. The best form is to select the fragment size as equal to the maximum noise size, which enables one to distribute the noise uniformly over the image at the output. That fragment size will determine the dimensions of the transformation matrix.

Maximal attenuation of the pulse noise amplitude requires a uniform distribution of the noise over the image as a result of the reverse transformation at the receiving end. This enables one to restore the information on corrupted or lost fragments. A problem arises over the form of the transformation matrix $A$ that minimizes the noise amplitude in the restored image.

Formula (5) defines the noise level $\Delta^{\prime}$ in the restored image in the case of one-sided strip transformation. If matrix $A$ is symmetrical and orthogonal, we have

$$
\Delta^{\prime}=A \Delta
$$

The analogous equation for two-sided transformation is derived from (8) as

$$
\Delta^{\prime}=A \Delta A
$$

We assume that the noise acting in the communication channel distorts only one image fragment (single pulse noise), which means only one of the components of the block vector $\Delta$ in (9) or the block matrix $\Delta$ in (10) can be different from zero. In both cases, the noise level $\Delta^{\prime}$ in the reconstructed signal will be determined by the element maximum in modulus of the orthogonal matrix $A$; in fact, if in (9) we assume $\Delta_{1}=1, \Delta_{2}=\ldots=\Delta_{N}=0$, then we get $\Delta^{\prime}=A_{1}$, where $A_{1}$ is the first column of matrix $A$. Then the amplitude of the noise $\Delta^{\prime}$ will be equal to the element maximum in modulus in the first column of matrix $A$ (in the general case, the entire matrix $A$ ).

Similarly, if we assume for example in (10) that $\Delta_{11}=1$ and put all the other $\Delta_{i j}=0$, we get

$$
\Delta^{\prime}=A_{1} \cdot A_{1}^{\mathrm{T}}=\left[a_{1 i} \cdot a_{1 j}\right]_{1}^{n} .
$$

In particular, for $n=3$ we get

$$
\Delta^{\prime}=\left[\begin{array}{lll}
a_{11} & a_{12} & a_{13} \\
a_{12} & a_{22} & a_{23} \\
a_{13} & a_{23} & a_{33}
\end{array}\right]\left[\begin{array}{lll}
1 & 0 & 0 \\
0 & 0 & 0 \\
0 & 0 & 0
\end{array}\right]\left[\begin{array}{lll}
a_{11} & a_{12} & a_{13} \\
a_{12} & a_{22} & a_{23} \\
a_{13} & a_{23} & a_{33}
\end{array}\right]=\left[\begin{array}{l}
a_{11} \\
a_{12} \\
a_{13}
\end{array}\right]\left[a_{11} a_{12} a_{13}\right] .
$$

Then the maximal element of matrix $\Delta^{\prime}$ will be $a_{\mathrm{m}}^{2}$, where $a_{\mathrm{m}}$ is the element maximal in modulus for the first column of matrix $A$. For an arbitrary position of the nonzero element in matrix $\Delta$, we get that $a_{\mathrm{m}}$ is the element maximal in modulus in matrix $A$.

As the purpose is maximally to attenuate the noise amplitude, in both cases we get a task of searching in the class of orthogonal matrices for that matrix in which the element maximal in modulus is minimal. A familiar solution relates to the 
case of $n$ a multiple of 4, namely normalized Hadamard matrices. Less familiar is the solution for even $n$ not a multiple of 4, the so-called conference matrices. In each of these, there is a zero diagonal, and the other elements are equal to \pm 1 .

Hadamard matrices ideally distribute uniformly single pulse noise over the entire area of the image while reducing the amplitude by a factor $n$ (if $m \neq n$, by a factor $\sqrt{m n}$ ). The above $C$ matrices are only slightly inferior and provide an attenuation of the noise by a factor $n-1$.

We are not aware of any general solution for odd $n$. As a result of lengthy searches, we have been able to identify only orthogonal matrices optimal in that sense for $n=3,5,7,9$, and 11 ; we restrict consideration to the case $n=3$, when the task has a clear-cut geometrical interpretation.

We temporarily lift the requirement of normalization for the columns of matrix $A$ and consider third-order matrices with pairwise orthogonal columns $A_{1}, A_{2}, A_{3}$, whose elements do not exceed 1 in modulus:

$$
\left|a_{i j}\right| \leq 1, \quad\left(A_{i}, A_{j}\right)=0, \quad i \neq j, \quad i, j=1,2,3 .
$$

Amongst these matrices, we need to find the one in which the element minimal in modulus is maximal. Geometrically, this means that we wish to place within a cube of edge 2 and orthogonal three-dimensional cross (antitank mine) of maximal size. The centers of the cube and cross lie at the origin and allow the spatial rotation of the cross as a single whole. Then the semiaxes of the cross act as the column vectors of matrix $A_{i}$, while the elements of matrix $A$ are provided by the Cartesian coordinates of the ends.

If the cross is oriented on the coordinate axes, one gets unit matrix, which is the worst case from the viewpoint of noise distribution in the image. It may appear preferable to orient the cross on the three cube diagonals, but this violates the orthogonality condition (the angle between the diagonals of a cube is arc cos $(1 / 3)$, which constitutes about $70^{\circ}$ ).

An intermediate case is optimal, which leads to the matrix

$$
A=\left[\begin{array}{ccc}
-0.5 & 1 & 1 \\
1 & -0.5 & 1 \\
1 & 1 & -0.5
\end{array}\right] .
$$

It is a very close analog of the Hadamard matrix for $n=3$, being orthogonal and symmetrical, with the maximal element after multiplication by the normalizing factor $1 / 3$ becoming $2 / 3$.

Then even in the simplest case, the search for the optimal matrix causes certain difficulties. This geometrical interpretation has an analogy with the Procrustes myth, and the multidimensional form of this task may be called an orthogonal Procrustes task. A simpler task is known under that name in matrix theory [3] which has a certain relation to the present case. The analogous geometrical interpretation for classical Hadamard matrices is considered in [4].

We give a further optimal matrix (a very close analog of Hadamard matrix) for $n=5$ :

$$
A=\frac{1}{11}\left[\begin{array}{ccccc}
-2 & 3 & 6 & 6 & 6 \\
3 & 6 & -6 & 6 & -2 \\
6 & -6 & -3 & 2 & 6 \\
6 & 6 & 2 & -6 & 3 \\
6 & -2 & 6 & 3 & -6
\end{array}\right]
$$

It is also orthogonal and symmetrical, and its maximal element is $6 / 11$.

For applications, it is sufficient to restrict oneself to Hadamard and $C$ matrices by splitting up the initial image into an even number of fragments while on the vertical and horizontal.

Determining Critical Noise Multiplicity. We estimate the performance of the strip method in image transmission in terms of the noise amplitude attenuation factor. The maximal attenuation of single noise is provided by the use of Hadamard matrices. If the initial image is split up into $n^{2}$ fragments, then on two-sided strip transformation the noise at the 
output is defined by $\Delta^{\prime}=A \Delta A$. If $A$ is a normalized Hadamard matrix of order $n$, then single pulse noise $\Delta_{1}$ is uniformly distributed over the entire area of the image, which guarantees reduction in its amplitude by a factor $n$ :

$$
J=\max _{\Delta \in \Delta_{1}} \frac{\left\|\Delta^{\prime}\right\|}{\|\Delta\|}=\frac{1}{n} .
$$

Here $\Delta_{1}$ denotes the set of noise processes that corrupt only one out of $n^{2}$ fragments in the transmitted image (single pulse noise), while as the norm one uses [1] the noise amplitude (Chebyshev norm).

Clearly, the performance of the strip method will decrease as the noise multiplicity increases, and for a certain multiplicity $r$, the coefficient $J$ attains the level of 1 or exceeds it. We call the minimum value of $r$ for which this occurs the critical noise multiplicity, while the strip transformation is ineffective for $J \geq 1$.

We give the solution for determining the critical noise multiplicity for two-sided strip transformation $Z=A X A$ on the assumption that $A$ is a normalized symmetrical Hadamard matrix of order $n$.

Let $\Delta \in \Delta_{k}$, where $\Delta_{k}$ is the set of all $k$-fold noise processes. We consider the case $k \leq n$ (the number of corrupted image fragments does not exceed the number of fragments in one row). Such noise corresponds to a matrix $\Delta$ containing $k$ nonzero elements denoted by $x_{1}, x_{2}, \ldots, x_{k}$; multiplication gives us the matrix $\Delta^{\prime}=A \Delta A$ with elements

$$
\Delta_{i j}^{\prime}=\left( \pm x_{1} \pm x_{2} \pm \ldots \pm x_{k}\right) / n
$$

We assume that the largest of the quantities $\left|x_{i}\right|$ is equal to 1 , and then none of the elements of matrix $\Delta^{\prime}$ will exceed 1 in magnitude; the maximum possible value of $\Delta_{i j}^{\prime}$ is attained for $x_{1}=x_{2}=\ldots=x_{k}=1$ and is equal to $k / n$. Then we get the attenuation coefficient as $J=k / n$. For $k=n$, it becomes 1 , so the critical noise multiplicity on two-sided transformation is $n$. We similarly consider one-sided strip transformation with a Hadamard matrix of order $N=n^{2}$ and find that in this case the critical multiplicity of the noise is again $n$ (the square root of the size of the Hadamard matrix). If on the other hand the one-sided or two-sided transformation is performed not with a Hadamard matrix but with some other, then the critical noise multiplicity is reduced.

We now determine the critical noise multiplicity for two-sided transformation with an orthonormalized $C$ matrix of sixth order:

$$
A=\frac{1}{\sqrt{5}}\left[\begin{array}{cccccc}
0 & 1 & 1 & 1 & 1 & 1 \\
1 & 0 & 1 & -1 & -1 & 1 \\
1 & 1 & 0 & 1 & -1 & -1 \\
1 & -1 & 1 & 0 & 1 & -1 \\
1 & -1 & -1 & 1 & 0 & 1 \\
1 & 1 & -1 & -1 & 1 & 0
\end{array}\right] .
$$

We consider fivefold interference of the form $\Delta=\operatorname{diag}\left(x_{1}, \ldots, x_{5}, 0\right)$, where $\left|x_{i}\right| \leq 1$.

The maximum possible amplitude after transformation is

$$
M=\max _{x_{1} \ldots x_{5}} \frac{1}{5}\left|\sum_{i=1}^{5} x_{i}\right| .
$$

The maximum $M=1$ is attained with $x_{1}=\ldots=x_{5}=1$, and in that case noise attenuation is not produced after transformation. On the other hand, for fourfold noise we get $M<1$, so the critical noise multiplicity is 5 .

Strip transformation with $C$ matrices gives the critical noise multiplicity as $n-1$, where $n$ is the order of the $C$ matrix. This is less by unity than for transformation with Hadamard matrices. 
Root Images in Two-Sided Strip Transformation. Let the initial image $X$ be split into $n \times n$ identical parts, for which we perform a two-sided strip transformation in accordance with

$$
Y=A X A,
$$

where $A$ is an orthogonal matrix, e.g., a normalized Hadamard matrix.

We consider identifying images that are invariant under (11), i.e., which are converted to the same images (apart from a constant factor):

$$
A X A=\lambda X .
$$

Such images are called proper ones or root images for the (11) transformation. It is an important task to define the root images for a given matrix $A$. If the noise in the communication channel coincides with the root image for the transformation used, then it remains unchanged on inverse transformation, and the effect of spreading out the noise over the image is not obtained. When one seeks root images, one can assume that the elements of matrix $X$ are not fragments of the initial image but instead real numbers. This simplifies the research and leads to the following task in linear algebra.

Root Matrix Problem. For a given nondegenerate square matrix $A$, we have to derive all the matrices $X$ and numbers $\lambda$ satisfying (12).

That formulation resembles the algebraic problem of the eigenvalues of matrix $A$, so we will call the pair $(\lambda, X)$ the root number and root matrix for matrix $A$. We derive a solution for orthogonal matrices $A$.

We first determine the root number $\lambda$. We transfer in (12) to determinants:

$$
|A| \cdot|X| \cdot|A|=\lambda^{n}|x| \Rightarrow \lambda^{n}=|A|^{2} \text {. }
$$

If matrix $A$ is orthogonal, then $|A|= \pm 1$ and $\lambda^{n}=1$. Consequently, we have $n$ root numbers $\lambda_{1}, \ldots, \lambda_{n}$, equal to the roots of 1 . All of them lie on units circled in the complex plane. Among them only two are real: $\lambda=1$ (image unaltered) and $\lambda=-1$ (image replaced by a negative). There are no other real roots. Further, if matrix $A$ is orthogonal and symmetrical (the case subsequently considered), the values $\lambda= \pm 1$ exhaust the set of root numbers. This is implied by the following theorem:

Theorem 1. The minimal polynomial for an orthogonal symmetrical matrix $A \neq \pm E$, no matter what its size, takes the form $\lambda^{2}-1$.

To prove this, it is sufficient to note that all the eigenvalues of an orthogonal matrix lie on unit circle, and all the eigenvalues of a symmetrical matrix are real. Together these conditions mean that some of the eigenvalues of matrix $A$ are 1 and the others are -1 . As a symmetrical matrix is diagonalizable, the minimal polynomial is $(\lambda-1)(\lambda+1)$. An exception is represented by unit matrix $E$ (where all the eigenvalues are 1) and also by the matrix $-E$ (where all the eigenvalues are -1 ). The minimal polynomials for these two matrices are correspondingly $\lambda-1$ and $\lambda+1$. In a symmetrical Hadamard matrix, the numbers of positive and negative eigenvalues are equal at $n / 2$. The corresponding eigenvectors form two orthogonally complementary subspaces of dimensions $n / 2$ each.

We now consider the root matrices for (11); the above proof implies that it is sufficient to consider the two cases following from (12) for $\lambda= \pm 1$ :

$$
A X A=X, \quad A X A=-X .
$$

We consider each of them.

Case $\lambda=1$. If matrix $A$ is orthonormal and symmetrical, then $A^{-1}=A$ and (2) becomes $A X=X A$. This means that matrices $A$ and $X$ are commutative. This implies that their eigenvectors coincide, and matrix $X$ can be represented as a polynomial in matrix $A$ :

$$
X=c_{1} E+c_{2} A+c_{3} A^{2}+\ldots+c_{m} A^{m-1}
$$

where $c_{i}$ are any real numbers, and $m$ is the order of the minimal polynomial for matrix $A$. 


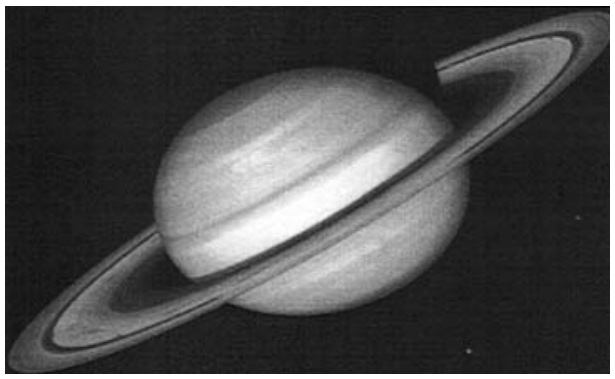

Fig. 2. Initial image of Saturn.

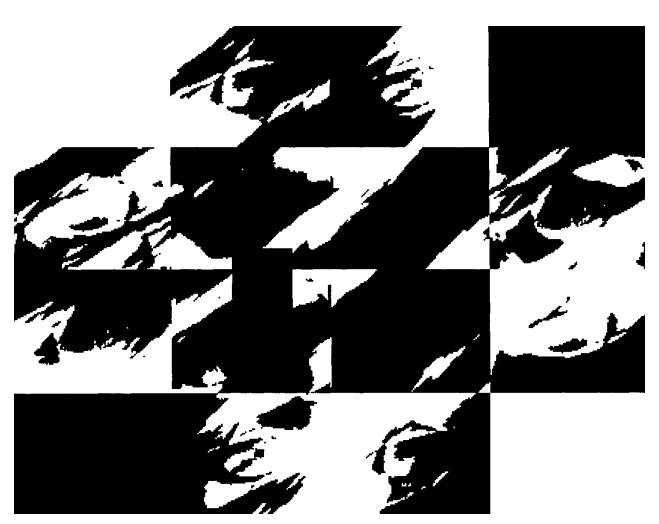

Fig. 3. Image of Saturn after two-sided strip transformation.

According to theorem 1, $m=2$ for orthogonal symmetrical matrices, so for them (13) becomes

$$
X=c_{1} E+c_{2} A
$$

The corresponding root matrices are linear combinations of unit matrix and matrix A. Formula (14) describes only some of the root matrices, which can be demonstrated on examples, while the general solution is given by the following theorem.

Theorem 2. Let $A$ be an orthogonal symmetrical matrix whose $m$ eigenvalues are 1, and let $H$ be the orthonormalized matrix of its eigenvectors. Then the general solution to the matrix equation $A X A=X$ contains $m^{2}+(n-m)^{2}$ arbitrary constants and takes the form

$$
X=H \tilde{X} H^{\mathrm{T}}, \quad \tilde{X}=\left[\begin{array}{cc}
C_{1} & 0 \\
0 & C_{2}
\end{array}\right],
$$

where $C_{1}$ and $C_{2}$ are any square matrices of dimensions $m \times m$ and $(n-m) \times(n-m)$, respectively.

To prove this, we write the initial matrix equation in the form $A X=X A$. This is a particular case of Silvester's equation [5]. To find the general solution, we reduce matrix $A$ to diagonal form by a similarity transformation:

$$
H^{\mathrm{T}} A H=E_{0}, \quad A=H E_{0} H^{\mathrm{T}}, \quad E_{0}=\left[\begin{array}{cc}
E_{m} & 0 \\
0 & -E_{n-m}
\end{array}\right],
$$

where $H$ is an orthonormalized matrix composed of the eigenvectors of matrix $A$ and $E_{m}$ is unit matrix of order $m$. 


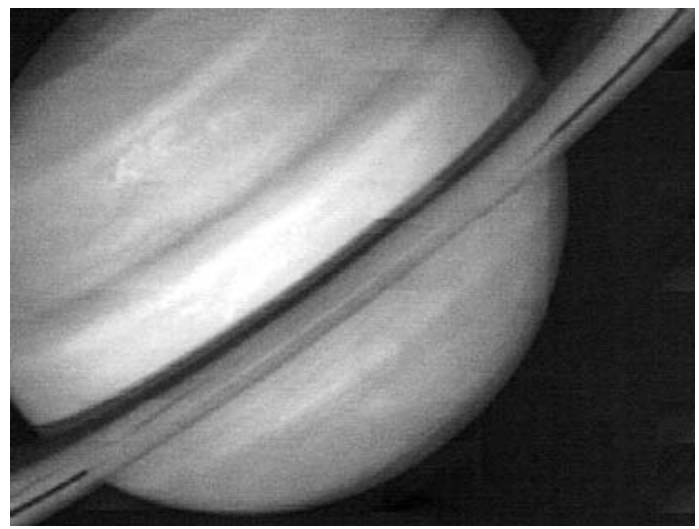

Fig. 4. Enlarged part of recovered image.

Then $A X=X A$ can be rewritten as

$$
H E_{0} H^{\mathrm{T}} X=X H E_{0} H^{\mathrm{T}}
$$

We multiply the two parts by $H^{\mathrm{T}}$ from the left and by $H$ from the right and put $\tilde{X}=H^{\mathrm{T}} X H$ to get

$$
E_{0} \tilde{X}=\tilde{X} E_{0}
$$

We represent matrix $\tilde{X}$ in block form $\tilde{X}=\left[\begin{array}{cc}\tilde{X}_{1} & \tilde{X}_{2} \\ \tilde{X}_{3} & \tilde{X}_{4}\end{array}\right]$, where the sizes of the diagonal blocks are $m$ and $n-m$. We perform the multiplication and equate blocks of the same name on left and right to get that $\tilde{X}_{2}=0, \tilde{X}_{3}=0$, i.e., $\tilde{X}=\left[\begin{array}{cc}\tilde{X}_{1} & 0 \\ 0 & \tilde{X}_{4}\end{array}\right]$; we return to the initial basis and put $C_{1}=\tilde{X}_{1}, C_{2}=\tilde{X}_{4}$, to get (15).

In that way, we have found the set of all root matrices $X$ corresponding to the root number $\lambda=1$.

Case $\lambda=-1$. The complete set of root images is here described by the following theorem.

Theorem 3. Let $A$ be an orthogonal symmetrical matrix whose $m$ eigenvalues are equal to -1 , and $H$ an orthonormalized matrix composed of its eigenvectors. Then the general solution to the matrix equation $A X A=-X$ contains $m^{2}+(n-$ $m)^{2}$ arbitrary constants and takes the form

$$
X=H \tilde{X} H^{\mathrm{T}}, \quad \tilde{X}=\left[\begin{array}{cc}
0 & C_{1} \\
C_{2} & 0
\end{array}\right],
$$

where $C_{1}$ and $C_{2}$ are any square matrices of dimensions $m \times m$ and $(n-m) \times(n-m)$, respectively.

The proof is given as for theorem 2, except that on the right in (16) there appears a minus sign:

$$
E_{0} \tilde{X}=-\tilde{X} E_{0}
$$

This leads to the diagonal blocks of matrix $\tilde{X}$ becoming zero, and the matrix takes the form $\tilde{X}=\left[\begin{array}{cc}0 & \tilde{X}_{2} \\ \tilde{X}_{3} & 0\end{array}\right]$.

We have thus obtained a general form of matrix $\tilde{X}$ for $\lambda=-1$; as in the previous case, it contains $m^{2}+(n-m)^{2}$ arbi- 
trary parameters. Amongst the possible forms we have a permutation matrix with ones on the diagonal $\tilde{X}=\left[\begin{array}{lll}0 & & 1 \\ & . & \\ 1 & 0\end{array}\right]$; this corresponds to the root matrix $X=H \tilde{X} H^{\mathrm{T}}=\left[H_{n} \ldots H_{1}\right] \cdot\left[H_{1} \ldots H_{n}\right]^{\mathrm{T}}$, in which $H_{i}$ is eigenvector $i$ of matrix $A$.

This root matrix analysis shows that two-sided strip transformation with a symmetrical Hadamard matrix has a large set of root images, which is due to the high symmetry of Hadamard matrices written in normal form. In most cases, the root images inherit this symmetry.

To reduce the cardinality of the root image set (for example, on the basis of cryptographic specifications, shorthand, or data protection), one should use unsymmetrical forms of the Hadamard matrices and also abandon the conditions $A=B$ and $m=n$ adopted from considerations of economy in computing resources.

Example of Image Strip Transformation. We present the principle of two-sided strip transformation on a particular example of an image processed by means of the toolbox Image Processing in the MATLAB package [6].

The initial image $P$ is a photograph of the planet Saturn (Fig. 2). We perform a two-sided matrix transformation for $n=4$, for which we divide the photograph of Saturn into four parts along the vertical and also four parts in the horizontal. The resulting block matrix $X$ contains 16 fragments, and it is used in two-sided strip transformation by means of a fourthorder Hadamard matrix:

$$
Z=A X A / 4
$$

The transmitted image $Z$ (with noise) is shown in Fig. 3. Each of the 16 fragments bears information on all 16 fragments of the initial photograph. On transmission, to this image we added noise in the form of a white square with $40 \times 50$ pixels. On recovering the image by means of the formula $X^{\prime}=A Z^{\prime} A / 4$, the noise is uniformly distributed over the entire picture and becomes largely inappreciable. This can be judged from an enlarged part of the recovered image (Fig. 4), in which there are weak lines of the noise square in each of the 16 fragments. Here the noise is uniformly distributed over all 16 fragments, and its amplitude is reduced by a factor of 4 , which means that the recovered photograph is of improved quality.

Conclusions. It has been shown that the strip method, which was proposed previously for noise-immune transmission of one-dimensional signals, allows of natural generalization to the two-dimensional case. One-sided and two-sided forms of strip transformation have been examined, and the best transformation matrices have been selected, the critical noise multiplicity has been determined, the set of root images for the strip transformation has been found, and an illustration has been given of the scope for this method on the strip transformation of a particular image.

The research was supported by the Russian Fund for Basic Research (Grants Nos. 04-01-00-464A and 04-07-90-354).

\section{REFERENCES}

1. $\quad$ L. A. Mironovskii and V. A. Slaev, Izmer. Tekh., No. 1, 12 (2002).

2. $\quad$ L. A. Mironovskii and V. A. Slaev, Izmer. Tekh., No. 7, 3 (2006).

3. J. Golub and C. van Loun, Matrix Calculations [Russian translation], Mir, Moscow (1999).

4. $\quad$ A. I. Medyanik, Mat. Fizika, Analiz, Geometriya, 4, No. 4, 458 (1997).

5. $\quad$ F. R. Gantmakher, Matrix Theory [in Russian], Fizmatlit, Moscow (2004).

6. I. S. Selyakov, Analysis and Computer Simulation of Image Strip Transformation, MSc Thesis [in Russian], St. Petersburg (2005). 